\title{
IDENTITIES INVOLVING PRODUCTS OF NUMBER-THEORETIC FUNCTIONS
}

\author{
R. G. BUSCHMAN
}

Abstract. General number-theoretic identities which involve various multiplications are given. The Tatuzawa-Iseki identity which occurs in certain elementary proofs of the Prime Number Theorem is included as a special case.

For three different multiplications which are defined on the set of number-theoretic (arithmetical) functions we will use the notations

$$
\begin{aligned}
f \cdot g & \text { if }(f \cdot g)(n)=f(n) g(n), \\
f * g & \text { if }(f * g)(n)=\sum(f(k) g(m): k m=n), \\
f \# g & \text { if }(f \# g)(x)=\sum(f(k) g(x / k) ; 1 \leqq k \leqq x) .
\end{aligned}
$$

It can be shown that the \#-operation is not associative and not commutative, but the other two operations have both of these properties. Properties of the ring of number-theoretic functions with the *-operation are discussed extensively in [1].

We shall use the following notations for functions: $\mu$ for the Möbius function, $\nu_{0}$ for the constant function for which $\nu_{0}(n)=1, \epsilon$ for the identity function with respect to the *-operation, $l$ for the logarithm function, and $\Lambda$ for the von Mangoldt function for which $\Lambda(n)=\log p$ if $n=p^{m}$ and $\Lambda(n)=0$ otherwise. In this notation we have $\mu * \nu_{0}=\epsilon$ and $\mu * l=\Lambda$.

IDENTITy A. $f \#(g \# h)=(f * g) \# h$.

Direct rearrangement of the sums gives us

$$
\begin{aligned}
(f \#(g \# h))(x) & =\sum\left(f(k) \sum(g(m) h(x / k m): 1 \leqq m \leqq x / k): 1 \leqq k \leqq x\right) \\
& =\sum(f(k) g(m) h(x / n): k m=n, 1 \leqq n \leqq x) \\
& =((f * g) \# h)(x) .
\end{aligned}
$$

The left inversion formulas for the \#-operation can be obtained from Identity A; these follow from the Möbius inversion formulas for the *-operation. In Identity $\mathrm{A}$ if we let $f=\nu_{0}, g=\mu, a=\mu \# h$, then $h=\nu_{0} \# a$; if we let $f=\mu, g=\nu_{0}, b=\nu_{0} \# h$, then $h=\mu \# b$. Two examples of other consequences are

Received by the editors July 21, 1969.

A MS Subject Classifications. Primary 1043; Secondary 1008.

Key Words and Phrases. Tatuzawa-Iseki identity, rearrangement of sums, arithmetical functions, number-theoretic identity. 


$$
f \#(g \# h)=g \#(f \# h), \quad(a \# b) \#(c \# d)=c \#((a \# b) \# d) .
$$

If in this last identity we set $c=\nu_{0}$, we can obtain

$$
(a \# b) \# d=\mu \#\left((a \# b) \#\left(\nu_{0} \# d\right)\right) \text {. }
$$

Identity B. $a \#(l \cdot(b \# c))=(a * b) \#(c \cdot l)+(a *(b \cdot l)) \# c$.

If we use $l(x / k)=l(x / n)+l(n / k)$ along with a rearrangement of the sums, then

$$
\begin{aligned}
&(a \#(l \cdot(b \# c)))(x) \\
&= \sum(a(k) l(x / k)(b \# c)(x / k): 1 \leqq k \leqq x) \\
&= \sum(a(k) b(m) c(x / n)(l(x / n)+l(n / k)): m k=n, 1 \leqq n \leqq x) \\
&= \sum((a * b)(n)(c \cdot l)(x / n): 1 \leqq n \leqq x) \\
&+\sum((a *(b \cdot l))(n) c(x / n): 1 \leqq n \leqq x) \\
&=((a * b) \#(c \cdot l))(x)+((a *(b \cdot l)) \# c)(x) .
\end{aligned}
$$

An alternate form can be obtained by use of Identity A,

$$
a \#(l \cdot(b \# c))=a \#(b \#(c \cdot l))+a \#((b \cdot l) \# c) .
$$

One special case of interest is obtained by setting $a=\mu$ and $b=\nu_{0}$. Since $\epsilon$ is a left identity for the \#-operation we have

$$
\mu \#\left(l \cdot\left(\nu_{0} \# c\right)\right)=c \cdot l+\left(\mu *\left(\nu_{0} \cdot l\right)\right) \# c .
$$

Since $\mu *\left(\nu_{0} \cdot l\right)=\Lambda$, the Tatuzawa-Iseki identity [2] which is used in some of the elementary proofs of the prime number theorem now follows;

$$
\text { if } d=l \cdot\left(\nu_{0} \# c\right), \quad \text { then } \mu \# d=c \cdot l+\Lambda \# c .
$$

If we set $a=\nu_{0}$ and $b=\mu$ and use $\nu_{0} *(\mu \cdot l)=-\Lambda$ we have analogously;

$$
\text { if } d=l \cdot(\mu \# c), \quad \text { then } \nu_{0} \# d=c \cdot l-\Lambda \# c \text {. }
$$

For another example consider Liouville's function $\lambda$ which is defined by $\lambda(n)=(-1)^{\Omega(n)}$ in which $\Omega(n)$ counts the total number of prime factors of $n$. Since $|\mu| * \lambda=\epsilon$ we have a number-theoretic identity analogous to the Tatuzawa-Iseki identity;

$$
\text { if } d=l \cdot(\lambda \# c), \quad \text { then }|\mu| \# d=c \cdot l+\Lambda^{\prime} \# c
$$

in which $\Lambda^{\prime}=|\mu| *(\lambda \cdot l)$ so that

$$
\begin{aligned}
\Lambda^{\prime}(n) & =\log p \quad \text { if } n=p^{2 m} \\
& =-\log p \quad \text { if } n=p^{2 m+1} \\
& =0 \quad \text { otherwise. }
\end{aligned}
$$


Similarly we can obtain

$$
\text { if } d=l \cdot(|\mu| \# c), \quad \text { then } \lambda \# d=c \cdot l-\Lambda^{\prime} \# c \text {. }
$$

Identity $\mathrm{B}^{\prime} . a \#(l \cdot(b \# c))=l \cdot((a * b) \# c)-(b *(a \cdot l)) \# c$.

This is closely related to Identity $B$ and it is derived in a similar manner, but by using $l(x / k)=l(x)-l(k)$. The Tatuzawa-Iseki identity and its analogs are again special cases.

\section{REFERENCES}

1. E. D. Cashwell and C. J. Everett, The ring of number-theoretic functions, Pacific J. Math. 9 (1959), 975-985. MR 21 \#7226.

2. T. Tatuzawa and K. Iseki, On Selberg's elementary proof of the prime-number theorem, Proc. Japan. Acad. 27 (1951), 340-342. MR 13, 725.

3. A. S. Amitsur, Some results on arithmetic functions, J. Math. Soc. Japan 11 (1959), 275-290. MR $26 \# 67$.

University of Wyoming, LaRamie, Wyoming 82070. 